\title{
Exploiting Interference Diversity for Event-Based Spectrum Sensing
}

\author{
Arash Parsa, Amin Aminzadeh Gohari, and Anant Sahai
}

\begin{abstract}
Spectrum sensing is a core problem in cognitive radio. Detecting the presence/absence of very weak primary users with a single antenna can be very difficult. Earlier it have been shown that uncertainties in the environment result in SNR walls that detectors cannot beat in a robust manner. Multiple antenna approaches show the potential of getting gains, but we show here that for a single user, multiple antenna detection still must suffer from an SNR Wall. The reason is that the real world uncertainty in noise is dominated by the potential presence of an unknown number of low-powered interference sources in the external environment. The traditional approach to collaborative spectrum sensing attempts to use the shadowing/multipath diversity across different users to boost the reliability of detection. We show here that there is another kind of diversity that is also available: interference diversity. This diversity captures the fact that these low-powered interference sources are local to individual users whereas the primary user has a global footprint. To exploit this diversity, we must shift our perspective from existence-based detection (whether the primary is present or not) to event-based detection (whether the primary has turned off or on). We study this and explore the limits to this approach.
\end{abstract}

\section{INTRODUCTION}

GPECTRUM sensing at very low SNR regimes has gained crucial importance in the context of cognitive radio. For example the required sensitivity in IEEE 802.22 standard is $-116 \mathrm{dBm}(-22 \mathrm{~dB}$ of signal to noise ratio) [1]. In addition to high sensitivity, the secondary users need to be able to operate in different settings and environments. Uncertainty in the noise statistics imposes fundamental limits on the sensitivity of secondary users. Below a certain level of SNR, detectors will not be able to sense the existence of the signal no matter how long they collect the data [2]. This level is called the SNR wall of the corresponding detector and partially quantifies the robustness of the

The authors are with the Department of Electrical Engineering and Computer Science at UC Berkeley. emails: \{parsa, aminzade, sahai\}@eecs.berkeley.edu
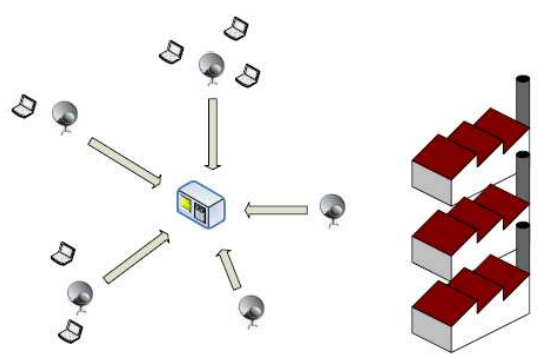

(4)

Fig. 1. Unintentional interferers (e.g. laptops in the figure) are sensed locally while the primaries are sensed globally.

system.

For this sensing problem different algorithms such as energy detection, matched filtering, cyclostationary feature detection [3] [4] [5] have been proposed. In this paper, we restrict ourselves to the scenarios in which one does not have knowledge about the features of the primary signal.

One can categorize these algorithms into single, non-cooperative multiple antenna and cooperative multiple antenna systems. From the first two categories we will briefly review radiometer and max-min eigenvalue detector [6] and discuss their fundamental limitations. The last category of algorithms, i.e. cooperative sensing algorithms, aim to increase the performance of detection of the overall system by using a network of sensors and making a decision based on the information gathered from different sensors. If sensors are experiencing independent shadowing or multipath fading effects, cooperative approach proves to be effective [8][7].

In this paper, we argue that one of the limiting factors in many of the proposed sensing algorithms is the choice of hypothesis. Many of the currently used algorithms choose to test the existence/absense of the primary signal. We refer to these detectors as "existence based" detectors. An equivalent set of hypothesis is to test enterance/exit/idle of the primary signal at any time instant. We refer to these detectors as "event based" detectors. When a set of events could be described using different sets of hypotheses, the choice that one makes greatly in- 
TABLE I

LIST OF A FEW NONCOHERENT DETECTION ALGORITHMS

\begin{tabular}{|c|c|}
\hline Detector & Characteristics \\
\hline Radiometer & $\begin{array}{c}\text { single-antenna; } \\
\text { non-cooperative }\end{array}$ \\
\hline Max-Min Eigenvalue [6] & $\begin{array}{c}\text { multi-antenna; } \\
\text { non-cooperative }\end{array}$ \\
\hline By Mishra et al. [7] & multi-antenna \\
By Ghasemi et al. [8] & cooperative \\
By Matsui et al [9] \\
The proposed detector
\end{tabular}

fluences the structure and performance of the corresponding hypothesis-testing algorithms. Whereas an existence/absense detector such as radiometer bases its decision on the expectation of different levels of energy under different hypothesis, an enterance/exit/idle detectors bases its decision on the expectation of local surges/local drops/small changes in the energy level of the received signal. A radiometer fires a false alarm when the average energy of the noise is high whereas a single-antenna enterance/exit/idle detector fires a false alarm when there are local surges or drops in the energy level of the noise. The latter event could happen when for example a significant noise interferer, such as a laptops leaking energy outside its permitted bandwidth, turns on or off.

Noise interferers are either "unintended emitters" (e.g. a laptop leaking energy outside its permitted bandwidth during a communication, or electronic circuits of a printer radiating electromagnetic waves during its activity) and "intended emitters" (other secondary networks using the bandwidth when the primary is absent). A sensor may have some knowledge about the possible turn on/turn offs of the intended emitters, but there is little it can do about the "unintended emitters".

In order to deal with the false-alarm events caused by unintended interferers, the following cooperative multi-sensor algorithm is proposed: when a primary signal enters or exits the system, it does so in a global scale causing many sensors to fire an alarm; however when an unintended emitters enters or exits the system, it does so in a geographically local scale triggering only a few neighboring sensors. The system can thus use this "Interference Diversity" to distinguish between the two events by gathering information from sensors placed at different physical locations(see figure 1 ).
Our work can be viewed in the context of "change detection" algorithms. Veeravalli [10] and Mei [11] study the application of quickest detection theory to decentralized decision systems. The quickest detection [12] [13] refers to real-time detection of abrupt changes in the distribution of observed signals as quick as possible. The analysis and results based on this method mostly depends on the assumption that distributions are known and fixed after and before the transition points. Furthermore, Li et. al. [14] apply the theory of quickest detection to problem of sensing primary signals in cognitive radio. Authors assume noise distribution is constant and known while there is some uncertainty about the amplitude and phase of the primary signal. Since the current proposed methods using quickest detection are based on knowledge of distributions they fail to address and capture the problem of noise uncertainty and robustness. Specifically many of the changes in the distribution of measured signal could be a result of entrance/exit of interferers and do not necessarily comply to any probabilistic model.

This paper is organized as follows. In Section II, sources of noise uncertainty are mentioned. As a prelude to its following sections, Section III reviews a couple of existence based sensing algorithms. The role of certain factors such as external interferers in imposing fundamental limits on the performance of this class of algorithms is highlighted. In Section IV, the new cooperative sensing algorithm is introduced and analyzed at an intuitive level; the rigorous formulation and analysis is moved to appendix B. Section $\mathrm{V}$ includes the simulation results. Lastly, appendix A includes the proof for a result on the SNR Wall for the max-min eigenvalue detector.

\section{Noise Uncertainty}

The sources of noise uncertainty can be decomposed into two categories: internal noise and external noise. An internal noise is a noise that is not inherently present in the environment but is generated by the detector itself. An external noise on the other hand is generated by interferers in the environment and is picked up by the detector.

While the uncertainty in the variance of the aggregate internal noise can be significantly reduced by calibrating the device in a controlled environment. The external noise coming from unintended emitters can not be calibrated because it depends on the environment in which the device is operating. The existence and the structure of the signals emitted by these in- 
terferers varies with location and time, and is thus not known apriori. The maximum received energy from the interferers is a lower bound on the noise uncertainty. Intuitively the overall uncertainty is equal to the maximum number of significant interferers that might co-exist in an environment times the average energy of a typical significant interferer.

\section{Existence Based Detectors}

In this section, we briefly review a class of detectors that are based on the following two hypothesis:

$\boldsymbol{H}_{0}$ : only noise $x[n]=w[n]$

$\boldsymbol{H}_{1}$ : signal + noise $x[n]=w[n]+r[n]$ where $x[n], w[n]$ and $r[n]$ are the received signal, the input noise and the primary signal at time $n$. We refer to these detectors as "existence based" or $H_{0}-H_{1}$ detectors.

The algorithms based on the above two hypothesis are usually designed for a fixed observation window. One hopes that by increasing the length of observation window, the algorithm will have a better performance. However the SNR wall analysis have shown that there are fundamental limitations to performance of the algorithm in presence of noise uncertainty [2]. The fundamental limits of these detectors, such as SNR Walls, are usually analyzed under the assumption that the detection time window approaches infinity.

As a prelude to the "Event Based Detectors" discussed in the next section, we review a couple of factors imposing limits on the performance of "Existence Based" detection algorithms. Of particular importance are the external interferers: in a practical environment there are interferers that may fool a detector who is looking for weak primary signals. The goal of the following subsections is partly to highlight the role of interferers in the performance of detection algorithms at low SNRs. We make this effect explicit in two particular detection algorithms: energy detector (radiometer), and maximum-minimum eigenvalue detector. The former detector has only one antenna while the latter has multiple antenna.

\section{A. Single Antenna Energy Detection: Radiometer}

An energy detector (radiometer) measures the average received energy in a time window, and makes a decision by comparing it with a fixed threshold.

First assume that the variance of noise is perfectly known. Then no matter how weak the primary signal is, as long as the observation window, $N$, is large enough, the detector will be able to identify it with

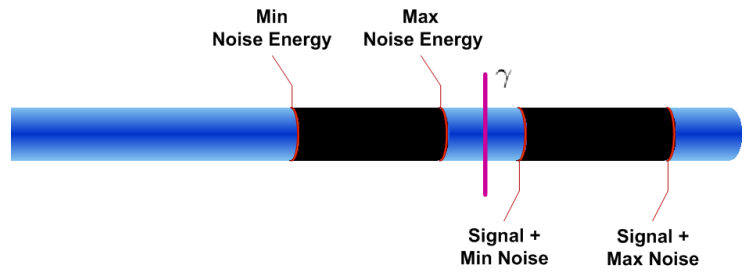

Fig. 2. Noise variance has a range from "min noise energy" to "max noise energy". When the signal is added, this interval shifts to higher values. If the signal is not strong enough and these two intervals collide, there would be no guarantee of success. Otherwise the detector is able to distinguish the two cases if it observes long enough.

(a)

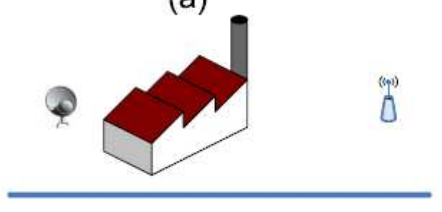

(b)

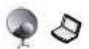

Fig. 3. Single antenna energy detector fails to distinguish weak primary from interferer.

arbitrarily high probability. In the case of radiometer, we can model the noise uncertainty by assuming that noise variance is known up to an interval. The length of this uncertainty interval determines the "SNR wall" for the radiometer (see figure 2). This analysis indicates that in terms of robustness one might prefer to have a larger average noise energy and less uncertainty rather than small average noise energy with much more uncertainty.

As mentioned in Section II, the SNR wall of the radiometer is no less than the maximum number of significant interferers that might co-exist in an environment times the average energy of a typical significant interferer (see figure 3).

\section{B. Max-Min Eigenvalue Detection}

In this section, we specifically discuss a multi antenna sensing algorithm known as Max-Min Eigenvalue Detector introduced by Liang and Zeng in [6] and highlight the effect of external interferers on the performance of this detector.

Liang and Zeng assume noise to be white and uniform (in terms of power) over different antennas. The authors argue that if primary signal does not exist, the correlation between samples at different anten- 
nas and/or different times will be zero. On the other hand, if primary signal is present, some of these correlations will not be zero. Therefore the detector can distinguish between these two scenarios by measuring the deviation of a covariance matrix from the identity matrix in terms of the ratio of maximum and minimum eigenvalues.

With the assumption of white and uniform (in terms of power) noise over different antennas and/or at different times, the max-min eigenvalue detector can overcome noise uncertainty. However, in a realistic scenario interferers are a major source of noise uncertainty and can not be ignored. Being generated by unknown sources such as electronic devices leaking outside their permitted bandwidth, the unintended interference noise may not be white and uniform (in terms of power) over different antennas. In other words from the perspective of max-min eigenvalue detector there is no difference between a very weak primary and an interferer. In the appendix we make this point more explicit by proving the following theorem on the SNR wall of this detector (see Appendix for notations):

Theorem A.1. For any positive $\epsilon$, there exists a threshold $\rho$ such that whenever the primary power $P$ is less than $\rho$, the detector can not guarantee a probability of miss-detection less than $1-\epsilon$. Furthermore, given the probability of false alarm $\operatorname{Pr}_{F A}, \rho$ is bounded from below by $\frac{(\gamma-1) \sigma_{\eta}^{2}}{c_{1}(\gamma+1)}$ where $c_{1}=O\left(\frac{1}{\epsilon}\right)$ and $\gamma=\operatorname{Thf}\left(\operatorname{Pr}_{F A}\right)$.

As we saw above, many of the known detectors are vulnerable to external interferers masquerading as weak primaries and vice versa. In Section IV, we propose an "event-based detection" algorithm for dealing with the negative effect of external interferers.

\section{Event Based Detection}

As discussed in Section III, sensing algorithms based on existence/absence $\left(H_{0}-H_{1}\right)$ hypothesis are vulnerable to external interferers and uncertainty about the environment. The $H_{0}-H_{1}$ hypothesis setting does no allow the sensing algorithm to exploit the fact that primary signals are sometimes present and sometimes absent. Intuitively, the transition between the existence/absence states of the primary signal creates rapid changes in the level of energy at the sensors. We refer to these changes as "energy edges"; if there is a surge in the energy level, we call it a "positive edge", otherwise we call it a "negative edge". For every time instance, we define three types of event

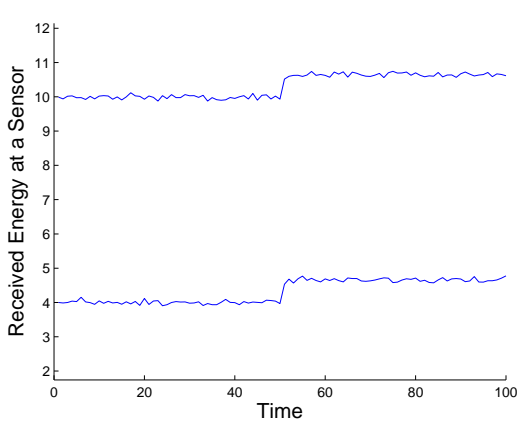

Fig. 4. Two sample noisy signal observation from the first noise model described in the "High Level Intuition" Section. Radiometer is very sensitive to uncertainty about the level of energy.

hypotheses:

$\int \mathbf{H}_{-}[n]:$ when there is a negative edge at time $n$; $\mathbf{H}_{+}[n]:$ when there is a positive edge at time $n$; $\mathbf{H}_{I}[n]$ : otherwise.

The new set of hypothesis may be thought of as the first derivative of the previous hypotheses. Knowing the location of these events is equivalent to knowing the existence/absence of the primary signals. In this part of the paper we show that by changing our hypotheses from sensing the level of energy to sensing the energy edges, we will be able to significantly improve our performance. Since this kind of sensing algorithm aims at detecting the enterance/exit of the primary signals and infer their existence from this information, we call it "event based sensing".

\section{A. High Level Intuition}

In this section, we would like to demonstrate the core ideas behind the new detection algorithm.

We start from a simplistic scenario in which the noise power is unknown but constant over time. Also assume that when the primary enters/exits, the received power changes instantaneously. Furthermore assume that in the time scale of interest, the primary changes its state at most once. If the signal power is less than the noise uncertainty (that is the range of the noise power) the radiometer fails. On the other hand, no matter how weak the primary signal is, an edge detection algorithm will be able to sense the primary if the observation window is long enough; and therefore no SNR Wall exists (see figure 4).

Next, consider the less restricted scenario in which the noise is comprised of different interferers each of which have constant power over time but are allowed to enter/exit (or turn on/off) ${ }^{1}$. Furthermore, we as-

${ }^{1}$ For instance this noise model may be used in the following 
sume that whenever an interferer enters or exists, the energy level changes instantaneously. Under this model, any single antenna edge detector will also fail since it can not distinguish between the entrance/exit of primary and that of interferers. To solve this problem, first we make the key observation that in many realistic scenarios interferers are short-range while primary signals are long-range (local-global assumption). Based on this assumption we propose a cooperative sensing scheme that can overcome the interference problem. The local-global assumption means that enterance/exit of the primary signal is sensed by most of the sensor nodes while interferers will only affect one or at most a few of the sensors. Therefore the system can detect the change in the status of primary whenever many sensors simultaneously declare so. It is important to note that we are proposing this sensing algorithm for a very low SNR regime. We will prove that as long as we do not deviate significantly from the local-global assumption, under certain conditions, the proposed algorithm is able to correctly detect weak primary signals.

\section{B. Formulation and Analysis}

In this section we explain the detection algorithm and its analysis at an intuitive level. For a more rigorous formulation and analysis of the system see Appendix B.

Consider a network of sensors geographically distributed in an area. Assume that the sensors are connected to a Central Unit $(C U)$ through communication links that have limited capacity. Each sensor node transmits its belief about a possible change in the status of primaries (i.e. whether a primary has entered (or exited), or that nobody has changed its state). At each time instant, the $C U$ will decide whether the primary has changed its state by, roughly speaking, taking a majority vote of the received messages from the sensors (see appendix B for a rigorous formulation).

The objective of a sensor is to detect the changes in the level of energy due to entrance/exit of the primary. One possible scheme is to compare the measured energy levels of the first half and second half of a shifting observation window. The goal of each sensor node is to provide the $C U$ with as much infor-

scenario: assume the interferer is a 802.11 wireless router leaking very weak signals out of its assigned bandwidth because of imperfect filters. Because of On/Off nature of the packet transitions there will be an entrance/exit pattern of interference in the band of interest.

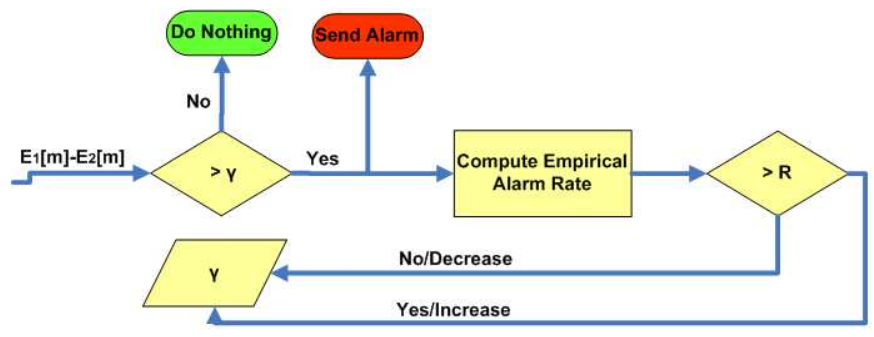

Fig. 5. Adaptive adjustment of the threshold at the sensor, $\gamma$ is the threshold, $\mathrm{R}$ is the allowed data rate

mation about the changes in the energy level of the environment as possible. In reality, because of limited resources, every sensor is forced to send only the most significant changes. Inevitably the sensors have to choose some threshold $\gamma$ and send an alarm to the central unit when the energy changes by more than this threshold. The length of the observation windows is small enough to ensure that the changes in the energy levels are not coming from changes in the channel statistics. Figure 10 depicts a sample output of this edge detector to the entrance of primary signal.

Having a lower threshold means sending more alarms on average and vice versa. Therefore limited data rate between the sensors and central unit imposes a lower bound on $\gamma$. If the links are wireless, meeting these lower bounds imply saturating all the links by using all of the available bandwidth and power.

Figure 5 provides an adaptive approach for setting $\gamma$ at each sensor node. Figure 7 shows a simulation result on the tradeoff between $\gamma$ and probability of false alarm under a specific noise model.

In the following paragraphs we explain the results in Appendix B at an intuitive level. We first analyze the behavior of the algorithm under the assumption that the network consists of a large number of nodes and that the capacity of the links between each sensor nodes and the $C U$ is some constant $R$.

The Appendix quantifies the local-global assumption in mathematical terms. The local-global assumption imposes certain constraints on the noise signals observed at different nodes. It is important to note that the noise signals may not have statistical description and can vary arbitrarily in time. The constraints imposed are therefore not on the statistical distributions of the noise signals but on their aggregate average behavior in time or in space.

In a large network the chance of an interferer ex- 
iting the system at some node of the network is the same as the chance of an interferer entering the network. The local-global assumption states that the interferers are short-range. This means that any entrance or exit of an interferer affects only a small number of sensor nodes. As long as our assumptions hold, one can expect that when status of the primaries do not change, the number of sensors experiencing a surge in their energy level should be roughly equal to the number of sensors experiencing a drop in their energy level. This intuition is formally expressed as the second constraint on noise in definition B.5.1.

The local-global assumption not only states that the interferers are local, but also that the primaries are global. This means that a change in the status of a primary is heard by many sensors. Roughly speaking, when the primary signal is not very weak, its entrance should increase the number of the sensors who experience a rapid increase in their energy level. Similarly, a primary leaving the system should increase the number of sensors who experience a rapid decrease in their energy level. This intuition is formally expressed as the second constraint on the noise in definition B.5.2.

Finally, the first constraint in definition B.5.1. implies that when the primaries do not enter or exit, the chosen threshold is sufficiently high so that the number of alarms generated on average over time by each sensor node is bounded.

Below, we mention a few examples of the noise signals that satisfy the constraints of definition B.5.1 and definition B.5.2 for appropriate choice of parameters:

- When the noise (including interference noise) at different nodes are white, independently and identically distributed (i.i.d.) according to an unknown but continuous density function (such as Gaussian).

- When the noise signal at each node has two components: 1 . independent white noise at different sensor nodes, identically distributed according to an unknown continuous density function; 2. Independent Poisson processes at different nodes with an unknown and possibly varying, but bounded rate modeling the entrance/exit of the interferers. The Poisson processes modeling the exit of the interferers appear with negative sign and are expected to on average cancel out the effect of Poisson processes modeling the entrance of the interferers.

If the power of the primary is very low, it will not be able to excite enough receivers and hence the detec-

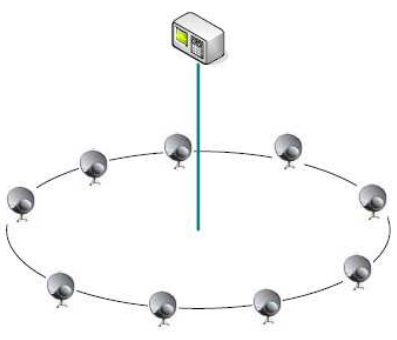

Fig. 6. In this example, $C U$ is placed at the top of the sensor nodes and at equidistance from all of them in the air

tion algorithm will fail. If the average energy of the primary signal at the sensor nodes is above a certain limit, the algorithm should however succeed. Theorem B.5.1. and its corollary formulates this intuition.

Roughly speaking, the minimum energy level required for detection is determined by the following factor: the lower the energy of the primary signal, the more sensitive the sensors need to be in detecting the changes in the energy level. But increasing the sensitivity of the sensor nodes, makes them vulnerable to entrance/exit of weaker interferers. This would not only increase the number of false alarms generated by each sensor node (and thus creating difficulty communicating them to the $C U$ ) but more importantly gradually invalidates the local-global assumption. A strong interferer may be heard by a larger group of sensor nodes. It should be however noted that the detection algorithm is robust in terms of the validity of the local-global assumption: for a large network, the second constraints of definition B.5.1 remains valid as long as each interferer affects only a sub-linear number of sensors i.e. the fraction of affected sensors is relatively small to the total number of sensors.

\section{"Computation in the air" method:}

Above, we assumed $R_{1}=R_{2}=\ldots=R_{m_{s}}=R$ allowing the total rate, $R_{1}+R_{2}+\ldots+R_{m_{s}}$ approach infinity. This is not a realistic assumption. We however note that the $C U$ only needs to know the total number of positive and the total number of negative alarms rather than the precise identity of those nodes who send positive or negative alarms. ${ }^{2}$ Consider the following strategy:

\footnotetext{
2 This is reminiscent of works which consider distributed computation combined with wireless communication (see for example [15]). It can be shown that separation between the communication stage and the computation stage turns is not always the optimal strategy, and a combined communication-computation scheme can improve the performance.
} 
The sensor nodes divide their time resources into consecutive intervals. In odd intervals, those terminals who want to transmit a positive alarm simultaneously transmit signals of power $P$ while in the even intervals those terminals who want to transmit a negative alarm simultaneously transmit signals of power $P$. These communications are taking place in a control channel which is different from the channel which needs to be sensed. The central unit, $C U$, measures the energy of the received signal (which is a superposition of the transmitted signals) in each of the intervals. These average energies are used to approximate the total number of sensors who are firing alarm; in particular the difference in the energy levels of consecutive odd and even intervals provides an estimate of the difference between the number of nodes who transmit positive alarms, and those who transmit negative alarms.

Consider the simplistic model ${ }^{3}$ shown in Figure 6 where $C U$ is equidistance from all of sensors and is experiencing the same channel to all of them. This makes sure that the sensor nodes are contributing equally to the total power received at the $C U$. If $\alpha$ fraction of the $m_{s}$ nodes are transmitting signals at power $P$, the expected value of the received energy at the $C U$ equals $\alpha . P . G_{a v}+P_{n}$ where $G_{a v}$ is the average gain from the sensor nodes to the $C U$ and $P_{n}$ is the average energy of the noise. Therefore the $C U$ can estimate $\alpha$ based on the average received energy. In the general scenario when the $C U$ is placed on the ground, the channels between the $C U$ and different sensor nodes would be different. And $C U$ would receive a weighted average of the transmitted signals from different sensors. Under the condition that the channels from the sensors to the $C U$ are different, if the sensor nodes have some knowledge about the strength of their channel to the $C U$, they can adjust their power correspondingly. Otherwise a more detailed investigation is required.

\section{Simulations}

The simulation results of this section are based on the following model: we consider a set of sensors uniformly distributed inside an square. At every point

\footnotetext{
${ }^{3}$ The result can be probably extended to the following model: assume that we divide the sensor nodes into the set of green nodes and red nodes. When the green nodes are talking, the red nodes are listening. The red nodes estimate the number of green nodes who believe that the primary has entered/existed and thus effectively playing the role of $C U$. We have thus in effect planted the $C U$ inside the sensor node themselves.
}

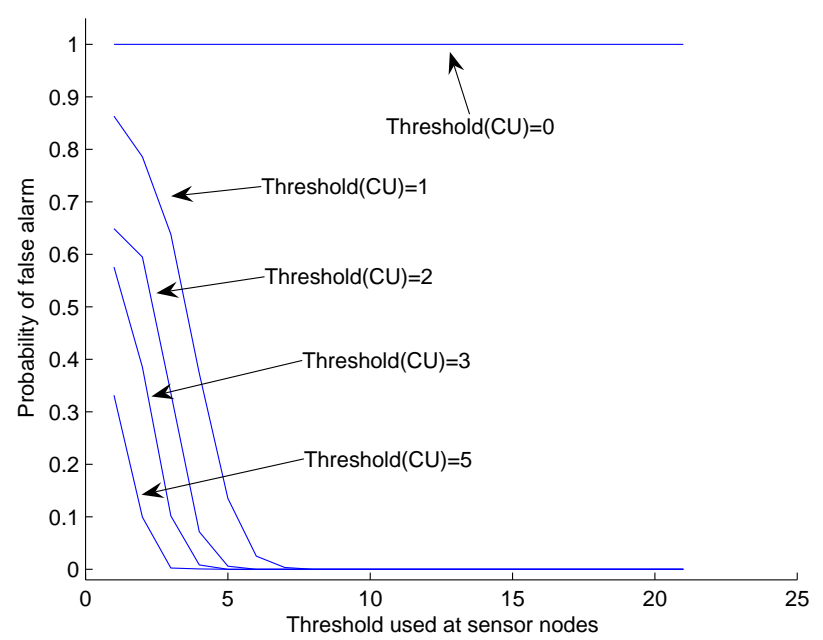

Fig. 7. The tradeoff between threshold and probability of false alarm

of the square lies an interferer that changes its status (i.e. turns on/turns off) with a fixed small probability. Around each sensor, we consider a smaller square. In this model, the interferers falling inside this square are sensed by the sensor without any attenuation.

Figure 7 shows the tradeoff between threshold and probability of false alarm. As expected, a decrease in the threshold increases the number of alarms generated by each sensors when the signal is not present, and thus the higher chance of $C U$ making a mistake.

Figure 8 depicts the trade off between number of sensors and the probability of false alarm. The goal of this simulation is two fold. Firstly, as long as the sensitivity area of sensors do not overlap, the alarms sent to $\mathrm{CU}$ are zero mean i.i.d. random variables. As we increase the number of sensors, their sum will be within an interval around zero with an increasing probability. Therefore probability of false alarm will be reduced. Secondly, we observe that increasing the number of sensors in a closed area will eventually make them sensitive to common interferers. In other words, in a dense network, the alarms sent by the sensors will become correlated and the performance would not be improved by the same trend.

\section{APPENDICES}

\section{A. SNR Wall Analysis for MaX-Min Eigenvalue Detector}

In this appendix, we prove that any interferer can impose limits on the performance of the max-min eigenvalue detector [6]. In this section, we adopt the same notation used in [6]. The authors implicitly as- 


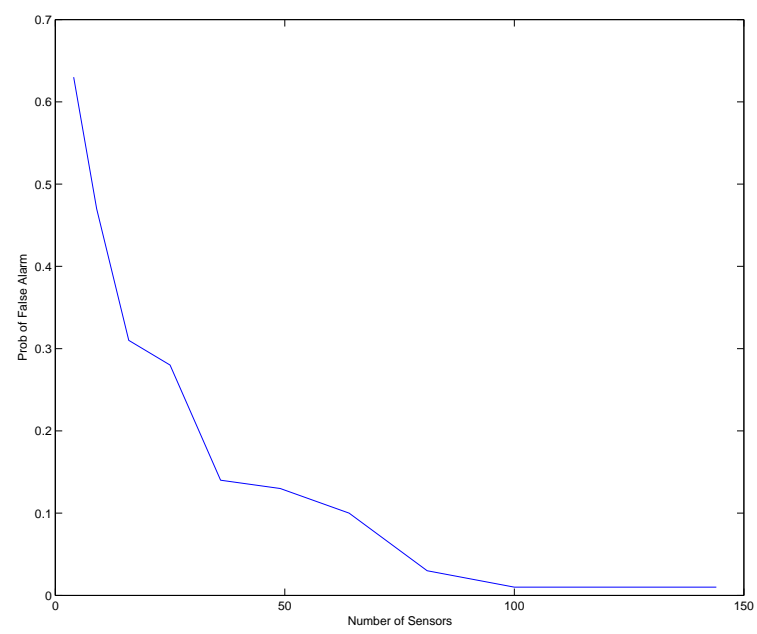

Fig. 8. Trade-off between number of sensors and probability of false alarm

sume that the coherence time of the multi-antenna receiver is infinity. We base our theorem on the same assumption.

Since the ratio $\frac{\lambda_{\max }\left(K_{\widehat{\mathbf{x}}}\right)}{\lambda_{\min }\left(K_{\widehat{\mathbf{x}}}\right)}$ can not be equal to one due to the existence of interferers even when the primary signal is not present, the detector has to set a threshold $\gamma$ and decide between the hypothesis $H_{0}$ and the hypothesis $H_{1}$ based on $\mathbf{1}\left[\frac{\lambda_{\max }\left(K_{\widehat{\mathbf{x}}}\right)}{\lambda_{\min }\left(K_{\widehat{\mathbf{x}}}\right)}>\gamma\right]$. The value of $\gamma$ would be uniquely determined by the probability of false-alarm.

Definition A.1. Assuming any specific model for noise, the threshold function "Thf" is the answer to the following equation: $\operatorname{Pr}\left(\frac{\lambda_{\max }\left(K_{\widehat{\mathbf{x}}}\right)}{\lambda_{\min }\left(K_{\widehat{\mathbf{x}}}\right)}>\right.$ $\left.\operatorname{Thf}\left(\operatorname{Pr}_{F A}\right)\right)=\operatorname{Pr}_{F A}$

Theorem A.1. For any positive $\epsilon$, there exists a threshold $\rho$ such that whenever the primary power $P$ is less than $\rho$, the detector can not guarantee a probability of miss-detection less than $1-\epsilon$. Furthermore, given the probability of false alarm $P r_{F A}, \rho$ is bounded from below by $\frac{(\gamma-1) \sigma_{\eta}^{2}}{c_{1}(\gamma+1)}$ where $c_{1}=O\left(\frac{1}{\epsilon}\right)$ and $\gamma=\operatorname{Thf}\left(\operatorname{Pr}_{F A}\right)$.

Proof: We prove the statement by contradiction. Fix some $P_{F A}$ and some $\epsilon>0$ and assume that the guarantee of $P_{M D}<1-\epsilon$ exists. In particular, the sensor should have $P_{M D}<1-\epsilon$ when the noise on the channels are white and uniform, and that $K_{\widehat{\mathbf{s}}}=P \mathbf{I}$. In this case, $K_{\widehat{\mathbf{x}}}=\kappa P H H^{\dagger}+\sigma_{\eta}^{2} \mathbf{I}_{M L}$ for some constant $\kappa$. Please note that intuitively if $P$ is small here, from the perspective of the detector it behaves like

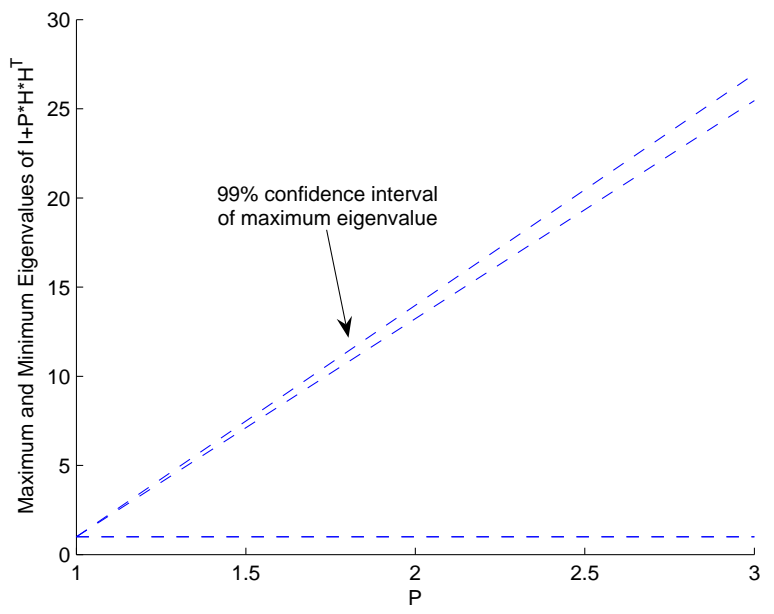

Fig. 9. Maximum and minimum eigenvalues of $I+p H H^{T}$ as a function of $p$, power of the signal. Minimum eigenvalue remains around one while maximum eigenvalue increases linearly. Here $H$ is a random matrix. At $p=0$, both eigenvalues are one. The maximum eigenvalue increases rapidly with the increase in $p$, while the minimum eigenvalue remains almost the same.

an interferer and thus the ratio between maximum and minimum eigenvalue should be close to one.

Using the fact that for any matrixes $A$ and $B$ we have:

$$
\left\{\begin{array}{l}
\lambda_{\max }(A+B) \leq \lambda_{\max }(A)+\lambda_{\max }(B) ; \\
\lambda_{\min }(A-B) \geq \lambda_{\min }(A)-\lambda_{\max }(B),
\end{array}\right.
$$

we can bound $\frac{\lambda_{\max }\left(K_{\widehat{\mathbf{x}}}\right)}{\lambda_{\min }\left(K_{\widehat{\mathbf{x}}}\right)} \quad$ from above by $\frac{\sigma_{\eta}^{2}+\kappa P \lambda_{\max }\left(H H^{\dagger}\right)}{\sigma_{\eta}^{2}-\kappa P \lambda_{\max }\left(H H^{\dagger}\right)}$ as long as $\sigma_{\eta}^{2}-\kappa P \lambda_{\max }\left(H H^{\dagger}\right)>0$.

In order to use Markov inequality in the above expression, we first need to show that $\mathrm{E}\left[\lambda_{\max }\left(H H^{\dagger}\right)\right]<$ $\infty$.

Using the Jensen inequality we have:

$$
\begin{gathered}
\mathrm{E}\left[\lambda_{\max }\left(H H^{\dagger}\right)\right] \leq \mathrm{E} \sqrt{\left.\sum_{i, j}\left(\mid H H^{\dagger}\right)_{i, j}\right|^{2}} \leq \\
\sqrt{\left.\mathrm{E} \sum_{i, j}\left(\mid H H^{\dagger}\right)_{i, j}\right|^{2}}<\infty
\end{gathered}
$$

Now, using Markov inequality, $\operatorname{Pr}\left(\lambda_{\max }\left(H H^{\dagger}\right) \leq\right.$ $\left.\frac{1}{\epsilon} \mathrm{E} \lambda_{\max }\left(H H^{\dagger}\right)\right) \geq 1-\epsilon$.

Let $c_{1}=\kappa \frac{1}{\epsilon} \mathrm{E} \lambda_{\max }\left(H H^{\dagger}\right)$. The above inequality implies that if $P<\frac{\sigma_{\eta}^{2}}{c_{1}}$ : 


$$
\begin{gathered}
\operatorname{Pr}\left(\sigma_{\eta}^{2}-\kappa P \lambda_{\max }\left(H H^{\dagger}\right)>0\right. \text { and } \\
\left.\frac{\sigma_{\eta}^{2}+\kappa P \lambda_{\max }\left(H H^{\dagger}\right)}{\sigma_{\eta}^{2}-\kappa P \lambda_{\max }\left(H H^{\dagger}\right)} \leq \frac{\sigma_{\eta}^{2}+c_{1} P}{\sigma_{\eta}^{2}-c_{1} P}\right) \geq 1-\epsilon
\end{gathered}
$$

Now, if $P<\frac{(\gamma-1) \sigma_{\eta}^{2}}{c_{1}(\gamma+1)}$, one can conclude that $\frac{\sigma_{\eta}^{2}+c_{1} P}{\sigma_{\eta}^{2}-c_{1} P} \leq \gamma$. This would in turn imply that

$$
\operatorname{Pr}\left(\frac{\sigma_{\eta}^{2}+\kappa P \lambda_{\max }\left(H H^{\dagger}\right)}{\sigma_{\eta}^{2}-\kappa P \lambda_{\max }\left(H H^{\dagger}\right)} \leq \gamma\right) \geq 1-\epsilon,
$$

and therefore

$$
\operatorname{Pr}_{M D}=\operatorname{Pr}\left(\frac{\lambda_{\max }\left(K_{\widehat{\mathbf{x}}}\right)}{\lambda_{\min }\left(K_{\widehat{\mathbf{x}}}\right)} \leq \gamma\right) \geq 1-\epsilon
$$

which is a contradiction.

\section{B. Formulation and Analysis of the Proposed Detection Algorithm}

\section{A. Model Configuration}

Consider a network of $m_{s}$ sensors, one Central Unit $(C U)$. The sensors are geographically distributed in an area. Furthermore each sensor has $m_{a}$ antennas. There are communication links between each sensor and the $C U$ with rate constraints $R_{1}, R_{2}, \ldots, R_{m_{s}}$. The message set at each node is (positive alarm, negative alarm, idle\}. A positive (or negative) alarm corresponds to the sensor's belief that the primary has entered (or exited) while an idle message corresponds to the belief that primary has not changed its state. At each time instant, the $C U$ will decide whether the primary has changed its state based on the received messages from the sensors. In addition, there is a latency constraint $D$ at the $C U$ meaning that the system needs to detect state transitions of the primary within delay $D$. Let $T$ be the minimum coherence time of all wireless communication channels. Assume that the minimum inter-transition duration of the primary signal is lower bounded by $T_{s}$, i.e. time between consecutive transitions of the primary is at least $T_{s}$.

We assume that there at most $m_{t}$ primaries. The active primaries are assumed to generate uncorrelated signals at any particular time instance.

For simplicity of analysis, we assume that whenever the primary signal enters/exits, all the sensor nodes detect, and transmit corresponding alarms which are received instantaneously by the $C U$. It should be however noted that in a realistic scenario there will inevitably be some delay for reasons such as the coding used by the sensor nodes, possible clock mismatches etc.
TABLE II

Notations

\begin{tabular}{|c|c|}
\hline Variable & Description \\
$C U$ & Central Unit \\
$m_{s}$ & Number of sensor nodes \\
$m_{a}$ & Number of antennas at each node \\
$m_{t}$ & Number of primaries \\
$R_{i}\left(i=1,2, \ldots, m_{s}\right)$ & Rate constraints \\
$D$ & Latency constraint at the $C U$ \\
$T_{s}$ & lower bound on the minimum \\
$T$ & inter-trans. duration of primaries \\
$\gamma_{j}$ & minimum coherence times \\
$\gamma_{c u}$ & Threshold at the $j^{t h}$ sensor \\
$\Phi_{j}().[m]$ & Threshold at the $C U$ \\
$\operatorname{Dt}(., .,).[\mathrm{m}]$ & Output of the $j^{t h}$ sensor \\
& Output of the $C U$ \\
\hline
\end{tabular}

\section{B. Detection Algorithm at the Sensors}

In this algorithm, the objective of a sensor is to detect the changes in the level of energy due to entrance/exit of the primary. One possible scheme is to compare the measured energy levels of the first half and second half of a shifting observation window. The length of the observation windows is chosen to be less than the minimum coherence time, $T_{c}$, ensuring that the changes in the energy levels are not coming from changes in the channel statistics. Other constraints on the duration of the observation window are the network's latency constraint, $D$, and the minimum inter-transition duration, $T_{s}$. The latter constraint guarantees that the primary signal is active during the second half of some observation window.

Definition B.2.1. For any positive $\gamma$, the output of the detection algorithm at the $j^{\text {th }}$ sensor $\Phi_{j}(\gamma)$ is defined as follows: assuming that the received signal at the $i^{t h}$ antenna of the $j^{\text {th }}$ sensor $\left(1 \leq i \leq m_{a}\right)$ is $x_{i, j}[$.$] , the sensor transmits:$

$\Phi_{j}(\gamma)[m]= \begin{cases}\text { Positive alarm } & \text { if } \mathbf{E}_{1, j}[m]-\mathbf{E}_{2, j}[m]>\gamma \\ \text { Negative alarm } & \text { if } \mathbf{E}_{1, j}[m]-\mathbf{E}_{2, j}[m]<-\gamma \\ \text { Idle message } & \text { otherwise }\end{cases}$ where

$\mathbf{E}_{1, j}[m]=\frac{1}{m_{a}} \sum_{r=1}^{m_{a}} \mathbf{E}_{2, j, r}[m]-\frac{1}{m_{a}} \sum_{r=1}^{m_{a}} \mathbf{E}_{1, j, r}[m]$, $\mathbf{E}_{2, j}[m]=\frac{1}{m_{a}} \sum_{r=1}^{m_{a}} \mathbf{E}_{1, j, r}[m]-\frac{1}{m_{a}} \sum_{r=1}^{m_{a}} \mathbf{E}_{2, j, r}[m]$, where

$\mathbf{E}_{1, j, r}[m]=\frac{1}{N_{0}} \sum_{n=0}^{N_{0}-1}\left|x_{r, j}\left[m-n-N_{0}-1\right]\right|^{2}$,

$\mathbf{E}_{2, j, r}[m]=\frac{1}{N_{0}} \sum_{n=0}^{N_{0}-1}\left|x_{r, j}[m-n]\right|^{2}$,

$2 N_{0}+1=\min \left(T_{s}, T_{c}, D\right)$.

We will discuss more about the choice of $\gamma$ in a 

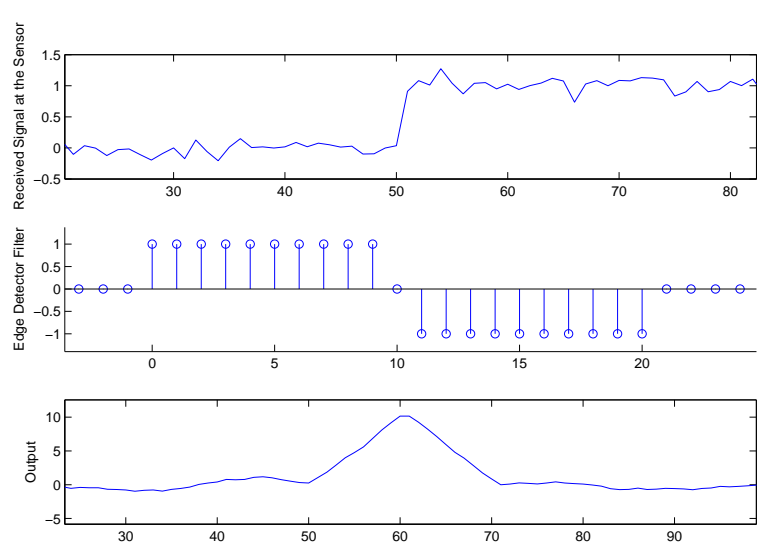

Fig. 10. The output of the edge detector filter will be approximately zero unless there is a surge or drop in the received energy level.

noisy environment later.

\section{Detection Algorithm at the Central Unit}

At any time instance $m$, the central unit receives signals from the sensor nodes. It seems intuitive for the $C U$ to take the majority vote. We proceed with this detecting strategy.

Definition B.3.1. For any positive $\gamma_{c u}$, the output of the detection algorithm at the $C U$ is defined as follows:

$$
\begin{aligned}
& \mathbf{D t}\left(\vec{\gamma}, \gamma_{c u}, \vec{R}\right)[m]= \\
& \left\{\begin{array}{l}
\mathbf{H}_{+}: \\
\frac{1}{m_{s}} \sum_{j=1}^{m_{s}} \mathbf{1}\left[\Phi_{j}\left(\gamma_{j}\right)[m]=\text { "Positive alarm" }\right]- \\
\frac{1}{m_{s}} \sum_{j=1}^{m_{s}} \mathbf{1}\left[\Phi_{j}\left(\gamma_{j}\right)[m]=\text { "Negative alarm" }\right]>\gamma_{c u} \\
\mathbf{H}_{-}: \\
\frac{1}{m_{s}} \sum_{j=1}^{m_{s}} \mathbf{1}\left[\Phi_{j}\left(\gamma_{j}\right)[m]=\right.\text { "Positive alarm"]- } \\
\frac{1}{m_{s}} \sum_{j=1}^{m_{s}} \mathbf{1}\left[\Phi_{j}\left(\gamma_{j}\right)[m]=\text { "Negative alarm"] }<-\gamma_{c u}\right. \\
\mathbf{H}_{I}: \\
\text { Otherwise }
\end{array}\right.
\end{aligned}
$$

where

$\vec{\gamma}=\left(\gamma_{1}, \gamma_{2}, \ldots, \gamma_{m_{s}}\right), \vec{R}=\left(R_{1}, R_{2}, \ldots, R_{m_{s}}\right)$.

\section{Analysis}

For simplicity of analysis, we assume that the channel is flat fading and that the primaries are using the Phase-shift keying (PSK) digital modulation, i.e. the constellation points chosen are positioned with uniform angular spacing around a circle.

The received signal at the $i^{\text {th }}$ antenna of the $j^{\text {th }}$ sensor is equal to $x_{i, j}[n]=\sum_{z=1}^{m_{t}} h_{i j}^{z} s_{z}[n]+w_{i, j}[n]$ where

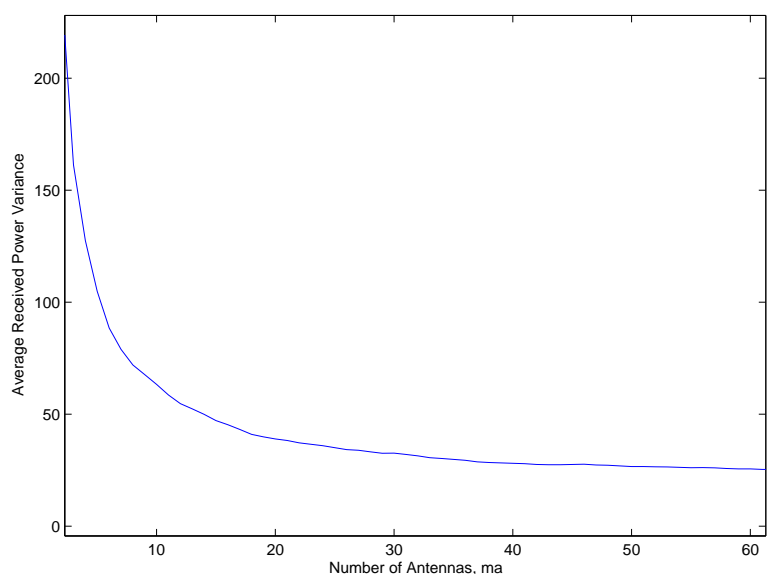

Fig. 11. Figure depicting the variance of $\frac{1}{m_{a}} \sum_{i=1}^{m_{a}}\left|w_{i, j}[n]\right|^{2}$ as a function of $m_{a}$. Here it is assumed that the noise signals is comprised of independent white Gaussian noises over different antennas, and also interference signals coming from an external interferer. As $m_{a} \rightarrow \infty$, the randomness coming from the white Gaussian noises and the fadings from the external interferer to the receiver decreases while the uncertainty in the energy level of the samples of the external interferer remains.

$h_{i j}^{z}$ is the channel gain and $s_{z}[n]$ is the signal transmitted by the $z^{\text {th }}$ primary. If the primary is not active, $s_{z}[n]=0$ otherwise it has constant amplitude $\left|s_{z}[n]\right|^{2}=P_{z}$.

We have:

$$
\begin{gathered}
\left|x_{i, j}[n]\right|^{2}=\sum_{z=1}^{m_{t}} \sum_{z^{\prime}=1}^{m_{t}} h_{i j}^{z} s_{z}[n]\left(h_{i j}^{z^{\prime}} s_{z^{\prime}}[n]\right)^{\dagger}+ \\
\left.\sum_{z=1}^{m_{t}} h_{i j}^{z} s_{z}[n] w_{i, j}[n]^{\dagger}+\sum_{z=1}^{m_{t}}\left(h_{i j}^{z} s_{z}[n]\right)^{\dagger} w_{i, j}[n]+\mid w_{i, j}[n]\right)\left.\right|^{2}
\end{gathered}
$$

Let $E_{*, j}[n]:=\frac{1}{m_{a}} \sum_{i=1}^{m_{a}}\left|x_{i, j}[n]\right|^{2}$.

Averaging over different antennas cancels the effect of multipath fading. Since the transmitted messages from different primaries at a time instance are uncorrelated we get the following equation using the law of large numbers:

$\lim _{m_{a} \rightarrow \infty} E_{*, j}[n]=\sum_{z=1}^{m_{t}} \rho_{j, z}[n] P_{z} \mathbf{1}\left[z^{\text {th }}\right.$ active at $\left.n\right]+$ $\left|W_{*, j}[n]\right|^{2}$.

where $\rho_{j, z}[n]$ is the average gain from the $z^{t h}$ transmitter to $j^{t h}$ sensor node and $\left|W_{*, j}[n]\right|^{2}$ is the average noise power at the different antennas at time instance $n:\left|W_{*, j}[n]\right|^{2}=\lim _{m_{a} \rightarrow \infty} \frac{1}{m_{a}} \sum_{i=1}^{m_{a}}\left|w_{i, j}[n]\right|^{2}$

Figure 11 depicts the average variance of $\frac{1}{m_{a}} \sum_{i=1}^{m_{a}}\left|w_{i, j}[n]\right|^{2}$ as a function of $m_{a}$ for a specific noise model. 
We therefore have: $\quad \lim _{m_{a} \rightarrow \infty} \mathbf{E}_{1, j}[m]=$ $\frac{1}{N_{0}} \sum_{n=0}^{N_{0}-1} E_{*, j}\left[m-n-N_{0}-1\right]$,

$\lim _{m_{a} \rightarrow \infty} \mathbf{E}_{2, j}[m]=\frac{1}{N_{0}} \sum_{n=0}^{N_{0}-1} E_{*, j}[m-n]$.

Observation B4.1. Since $2 N_{0} \leq T_{c}, \rho_{j, z}[n]$ does not change within an observation window. We can thus draw the conclusion that if the status of a primary does not change throughout the observation window, it does not contribute to the difference $\lim _{m_{a} \rightarrow \infty} \Phi_{j}(\gamma)[m]=\lim _{m_{a} \rightarrow \infty}\left(\mathbf{E}_{1, j}[m]-\mathbf{E}_{2, j}[m]\right)$.

In the following subsections, we analyze the detection algorithm under different configurations. In the first subsection, we analyze the asymptotic behavior of the algorithm under the assumption that $R_{1}=R_{2}=\ldots=R_{m_{s}}=R$. We will prove that the detector has no SNR-Wall when $m_{a}, m_{s} \rightarrow \infty$ as long as the noise is following from a certain model. The problem with this configuration is however that the total rate, $R_{1}+R_{2}+\ldots+R_{m_{s}}$ approaches infinity as $m_{s} \rightarrow \infty$.

In the next subsection, we justify the choice of $R_{1}=$ $R_{2}=\ldots=R_{m_{s}}=R$ arguing that the $C U$ only needs to know the total number of positive and the total number of negative alarms; $C U$ is only interested in this function of the transmitted messages, and does not need to know the identity of the nodes that are transmitting positive or negative alarms.

\section{D.1 Equal Rate At All Sensors}

Assume that $R_{1}=R_{2}=\ldots=R_{m_{s}}=R$. We will define a class of noise functions under which the detection algorithm has arbitrarily low probability of false alarm and miss-detection as $m_{s}$ and $m_{a}$ converge to infinity for arbitrarily weak primaries. The constraint imposed on the noise functions quantifies the local-global assumption according to which the noise signal observed at different sensors are independent.

Definition B.5.1. For any integer $N_{0}$ and vectors of positive real numbers $\vec{\gamma}$ and $\vec{R}$, Noise model $\Theta\left(\vec{\gamma}, \vec{R}, N_{0}, m_{s}, m_{a}\right)$ is the set of noise functions $w_{i, j}[n] \quad\left(1 \leq i \leq m_{a}, \quad 1 \leq j \leq m_{s}, \quad n=\right.$ $\ldots,-2,-1,0,1,2, \ldots)$ satisfying the following two constraints. Whenever primaries are absent, i.e. $x_{i, j}[]=$. $w_{i, j}[$.$] ,$

1. $\forall j$ :

$\lim _{M \rightarrow \infty} \frac{1}{M} \sum_{m=1}^{M} \mathbf{1}\left[\Phi_{j}\left(\gamma_{j}\right)[m] \neq\right.$ "Idle message" $] \leq R$ $2 . \forall m$ :

$\lim _{m_{s} \rightarrow \infty} \quad\left\{\frac{1}{m_{s}} \quad \sum_{j=1}^{m_{s}} \quad \mathbf{1}\left[\Phi_{j}\left(\gamma_{j}\right)[m] \quad=\right.\right.$
"Positive alarm"] -

$\frac{1}{m_{s}} \sum_{j=1}^{m_{s}} \mathbf{1}\left[\Phi_{j}\left(\gamma_{j}\right)[m]=\right.$ "Negative alarm" $\left.]\right\}=0$

Remark: The first constraint ensures that for the chosen threshold $\gamma_{j}$, the uncoded transmission of the alarms generated by the noise signal when primaries are absent do not violate the rate constraints. Observation B4.1 implies that when some of the primaries are present but do not change their state, the same alarms rate would be generated. Clearly increasing $\gamma_{j}$ makes enlarges the set of noise sequences that satisfy the first property. In the extreme case of $\gamma_{j}=\infty$, the constraint would be valid for all $R_{j}$. The second assumption is intuitively saying that noise is unbiased: as $m_{s} \rightarrow \infty$, the number of positive alarms and negative alarms generated by the noise converge together. This phenomenon would happen if the noise sequences are each unbiased and behave independently of each other. If the local-global assumption fails, the alarms generated by different nodes will no longer be independent of each other and this constraint may no longer be valid.

Definition B.5.2. For any constant $c$, Noise model $\Upsilon\left(c, \vec{\gamma}, N_{0}, m_{s}, m_{a}\right)$ is the set of noise functions $w_{i, j}[n] \quad\left(1 \leq i \leq m_{a}, \quad 1 \leq j \leq m_{s}, \quad n=\right.$ $\ldots,-2,-1,0,1,2, \ldots)$ satisfying the following two constraints. Whenever primaries are absent, i.e. $x_{i, j}[]=$. $w_{i, j}[$.$] , either of the following two conditions are sat-$ isfied:

$$
\begin{aligned}
& \delta_{1}=\inf _{m} \lim _{m_{s} \rightarrow \infty} \overrightarrow{\gamma^{\prime}: \gamma_{j} \geq \gamma_{j}^{\prime}} \text { for } \inf _{j=1,2, . . m_{s}} \text { and } \frac{1}{m_{s}}\left|\vec{\gamma}-\overrightarrow{\gamma^{\prime}}\right|_{1} \geq c \\
& \left\{\frac { 1 } { m _ { s } } \sum _ { j = 1 } ^ { m _ { s } } \left[\mathbf{1}\left[\Phi_{j}\left(\gamma_{j}\right)[m]=\text { "Positive alarm" }\right]-\right.\right. \\
& \left.\left.\mathbf{1}\left[\Phi_{j}\left(\gamma_{j}^{\prime}\right)[m]=\text { "Positive alarm" }\right]\right]\right\}>0
\end{aligned}
$$

or

$$
\begin{gathered}
\delta_{2}=\inf _{m} \lim _{m_{s} \rightarrow \infty} \overrightarrow{\widehat{\gamma}}: \gamma_{j} \leq \widehat{\gamma}_{j} \text { for } j=1,2, . . m_{s} \text { and } \frac{1}{m_{s}}|\vec{\gamma}-\overrightarrow{\widehat{\gamma}}|_{1 \geq c} \\
\left\{\frac { 1 } { m _ { s } } \sum _ { j = 1 } ^ { m _ { s } } \left[\mathbf{1}\left[\Phi_{j}\left(\gamma_{j}\right)[m]=\text { "Negative alarm" }\right]-\right.\right. \\
\left.\left.\mathbf{1}\left[\Phi_{j}\left(\widehat{\gamma}_{j}\right)[m]=\text { "Negative alarm" }\right]\right]\right\}>0 .
\end{gathered}
$$

Remark : Clearly for any $j$ and any $\gamma_{j}^{\prime} \leq \gamma_{j}$,

$$
\begin{gathered}
\mathbf{1}\left[\Phi_{j}\left(\gamma_{j}\right)[m]=\text { "Positive alarm" }\right] \geq \\
\mathbf{1}\left[\Phi_{j}\left(\gamma_{j}^{\prime}\right)[m]=\text { "Positive alarm" }\right]
\end{gathered}
$$


The first condition ensures that in the limit case, whenever the average gap between $\gamma_{j}^{\prime}$ and $\gamma_{j}$ is at least a constant $c$, the rate of positive alarm is sensitive to changes in $\gamma$. The second condition has a similar interpretation. The reason for introducing these two conditions is that intuitively when a primary enters the system, the chance of passing threshold $\gamma$, and thus of "Positive alarm", increases whereas the chance of passing $-\gamma$, and thus of "Negative alarm", decreases. This is because the effective threshold for "Positive alarm" from the perspective of noise decreases, whereas the effective threshold for "Positive alarm" from the perspective of noise decreases.

Theorem B.5.1. The detection algorithm $\operatorname{Dt}\left(\vec{\gamma}, \gamma_{c u}, \vec{R}\right)$ succeeds with probability one as $m_{a}, m_{s} \rightarrow \infty$ if

- The detector is operating in an environment with a noise sequence from $\Theta\left(\vec{\gamma}, \vec{R}, N_{0}\right) \cap \Upsilon\left(c, \vec{\gamma}, N_{0}, m_{s}, m_{a}\right)$;

- The average power of the primary that has entered/existed the system over all antennas of the system is at least $c$;

- $\gamma_{c u}<\max \left(\delta_{1}, \delta_{2}\right)$ where $\delta_{1}$ and $\delta_{2}$ are defined in $\Upsilon\left(c, \vec{\gamma}, N_{0}, m_{s}, m_{a}\right)$.

Proof. Assume that no primary changes its status in the interval $\left[n-N_{0}-1, n+N_{0}-1\right]$. The second property of $\Theta\left(\vec{\gamma}, \vec{R}, N_{0}\right)$ ensures that $\lim _{m_{s} \rightarrow \infty} \operatorname{Dt}\left(\vec{\gamma}, \gamma_{c u}, \vec{R}\right)=\mathbf{H}_{I}$.

Next assume that the $z^{\text {th }}$ primary turns on at time $n$. Since $2 N_{0}+1$ is less than the minimum intertransition duration of the primary signals, $T_{s}$, there can be no other primary arriving or leaving in the interval $\left[n-N_{0}-1, n+N_{0}-1\right]$. Thus, other primaries do not contribute to $\lim _{m_{a} \rightarrow \infty} \Phi_{j}(\gamma)[m]$ according to observation B4.1. Since $2 N_{0}+1$ is less than the coherence time, $\rho_{j, z}[n]$ is constant during the interval $\left[n-N_{0}-1, n+N_{0}-1\right]$; let $\rho_{j}:=\rho_{j, z}[u]$ (for $u \in\left[n-N_{0}-1, n+N_{0}-1\right]$ ).

The best opportunity to detect the entrance of the $z^{t h}$ primary is at time $n+N_{0}-1$, when the very beginning of the second half of the observation window is the entrance time of the $z^{\text {th }}$ primary. The average energy in the second part of the window is increased by $\rho_{j} P_{z}$. Let $\gamma_{j}^{\prime}=\gamma_{j}-\rho_{j} P_{z}, \widehat{\gamma}_{j}=\gamma_{j}+\rho_{j} P_{z}$. Since the average power of the primary that has entered/existed the system over all antennas of the system is at least $c$, we get $\frac{1}{m_{s}} \sum_{j=1}^{m_{s}} \rho_{j} P_{z} \geq c$. The condition imposed in $\Upsilon\left(c, \vec{\gamma}, N_{0}, m_{s}, m_{a}\right)$ ensures that the average number of positive alarms that the $C U$ receives is greater than the average number of nega- tive alarms by at least $\max \left(\delta_{1}, \delta_{2}\right)$. This means that the detector $\mathbf{D t}\left(\vec{\gamma}, \gamma_{c u}, \vec{R}\right)$ succeeds to detect the primary.

Corollary: The detector will be able to detect the entrance/exit of primary signal with average power $p_{\min }$ if noise signal belongs to the set:

$$
\bigcap_{c \geq p_{\min }} \Upsilon\left(c, \vec{\gamma}, N_{0}, m_{s}, m_{a}\right) \bigcap \Theta\left(\vec{\gamma}, \vec{R}, N_{0}\right)
$$

\section{REFERENCES}

[1] S. Shellhammer, Numerical Spectrum Sensing Requirements, IEEE Std. 802.22-06/0088r0, Jun. 2006.

[2] R. Tandra, A. Sahai, "SNR Walls for Signal Detection," IEEE Tran. Signal Process., vol. 2, NO.1, Feb. 2008.

[3] A. Sahai and D. Cabric, "Spectrum sensing: fundamental limits and n practical challenges," in Proc. IEEE International Symposium on New Frontiers in Dynamic Spectrum Access Networks (DySPAN), (Balti- 0.1 ,.more, MD), Nov. 2005.

[4] W. A. Gardner, "Spectral correlation of modulated signals: part i-analog modulation," IEEE Trans. Communications, vol. 35 , no. 6 , pp. 584-595, 1987.

[5] D. Cabric, S. M. Mishra, R. W. Brodersen, Implementation Issue in Spectrum Sensing for Cognitive Radios, The Thirty- Eighth Asilomar Conference on Signals, Systems and Computers, vol.1, pp. 772-776, November 2004.

[6] Y. Zeng and Y.C. Linag "Maximum-Minimum Eigenvalue Detection for Cognitive Radio", The 18th Annual IEEE International Symposium on Personal, Indoor and Mobile Radio Communications. (PIMRC07)

[7] S. M. Mishra, A. Sahai, and R. W. Broderson, "Cooperative sensing among cognitive radios," Proc. IEEE International Conference on Comunications (ICC) 2006, Istanbul, Turkey, Jun. 2006.

[8] A. Ghasemi and E. S. Sousa, Collaborative Spectrum Sensing for Opportunistic Access in Fading Environments, IEEE DySPAN 2005, Baltimore, USA, pp.131-136, 2005.

[9] Matsui, M. Shiba, H. Akabane, K. Uehara, K., "A Novel Cooperative Sensing Technique for Cognitive Radio," The 18th Annual IEEE International Symposium on Personal, Indoor and Mobile Radio Communications (PIMRC'07)

[10] V. V. Veeravalli, Decentralized quickest change detection," IEEE Trans. on Inform. Theory, vol.47, no.4, May 2001.

[11] Y. Mei, Information Bounds and Quickest Change Detection in Decentralized Decision Systems", IEEE Trans. on Inform. Theory, Vol. 51, no.7, July 2005.

[12] M. Basseville and I. Nikiforov, Detection of Abrupt Changes: Theory and Applications. Prentice-Hall, Englewood Cliffs, NJ, 1993.

[13] T. Kailath and H. V. Poor, Detection of Stochastic Processees, IEEE Trans. on Inform. Theory, Vol. 44, pp. 22302259 , Oct. 1998.

[14] H. Li, C. Li and H. Dai, Quickest Spectrum Sensing in Cognitive Radio, 2008 Conference on Information Sciences and Systems (CISS).

[15] B. Nazer and M. Gastpar, "Computation over MultipleAccess Channels," IEEE Transactions on Information Theory, Special Issue on Models, Theory, and Codes for Relaying and Cooperation in Communication Networks, vol.53, no.10, pp.3498-3516, October 2007. 